\title{
The predicted secretome and transmembranome of the poultry red mite Dermanyssus gallinae
}

\author{
Sabine Schicht ${ }^{1}$, Weihong $\mathrm{Qi}^{2}$, Lucy Poveda ${ }^{2}$ and Christina Strube ${ }^{{ }^{*}}$
}

\begin{abstract}
Background: The worldwide distributed hematophagous poultry red mite Dermanyssus gallinae (De Geer, 1778) is one of the most important pests of poultry. Even though 35 acaricide compounds are available, control of D. gallinae remains difficult due to acaricide resistances as well as food safety regulations. The current study was carried out to identify putative excretory/secretory (pES) proteins of $D$. gallinae since these proteins play an important role in the host-parasite interaction and therefore represent potential targets for the development of novel intervention strategies. Additionally, putative transmembrane proteins (pTM) of D. gallinae were analyzed as representatives of this protein group also serve as promising targets for new control strategies.

Methods: D. gallinae pES and PTM protein prediction was based on putative protein sequences of whole transcriptome data which was parsed to different bioinformatical servers (SignalP, SecretomeP, TMHMM and TargetP). Subsequently, pES and PTM protein sequences were functionally annotated by different computational tools.

Results: Computational analysis of the D. gallinae proteins identified 3,091 pES (5.6\%) and 7,361 pTM proteins (13.4\%). A significant proportion of pES proteins are considered to be involved in blood feeding and digestion such as salivary proteins, proteases, lipases and carbohydrases. The cysteine proteases cathepsin $D$ and $L$ as well as legumain, enzymes that cleave hemoglobin during blood digestion of the near related ticks, represented 6 of the top-30 BLASTP matches of the poultry red mite's secretome. Identified PTM proteins may be involved in many important biological processes including cell signaling, transport of membrane-impermeable molecules and cell recognition. Ninjurin-like proteins, whose functions in mites are still unknown, represent the most frequently occurring PTM.
\end{abstract}

Conclusion: The current study is the first providing a mite's secretome as well as transmembranome and provides valuable insights into D. gallinae pES and pTM proteins operating in different metabolic pathways. Identifying a variety of molecules putatively involved in blood feeding may significantly contribute to the development of new therapeutic targets or vaccines against this poultry pest.

Keywords: Next generation sequencing, In silico, Excretory/secretory proteins, Secreted proteins, Transmembrane proteins, Acari, Blood digestion, Embryogenesis, Ninjurin

\footnotetext{
* Correspondence: christina.strube@tiho-hannover.de

${ }^{1}$ Institute for Parasitology, University of Veterinary Medicine Hannover,

Buenteweg 17, 30559 Hannover, Germany

Full list of author information is available at the end of the article
} waiver (http://creativecommons.org/publicdomain/zero/1.0/) applies to the data made available in this article, unless otherwise stated. 


\section{Background}

The poultry red mite Dermanyssus gallinae (De Geer, 1778) is a worldwide distributed parasitic mite of poultry. It affects its hosts by blood feeding, causing skin irritations, weight loss, restlessness, feather pecking, and an increased incidence of cannibalism $[1,2]$. Furthermore, in cases with a high infestation rate it may even cause death due to anemia. As a consequence, the parasite leads to high economic losses in poultry farming with estimated annual costs of $€ 130$ million throughout the European Union alone. Therefore, the poultry red mite is the major pest for poultry farming $[2,3]$. The prevalence of $D$. gallinae depends on flock systems: infestation rates were $4 \%$ in cage systems but $33 \%$ in alternative systems and $67 \%$ of backyard flocks $[3,4]$. In different countries, D. gallinae prevalence rates can reach up to $80-90 \%$ as shown for the United Kingdom, The Netherlands, Italy, Serbia, Montenegro, Morocco and Japan [3]. Control of the poultry red mite is extremely difficult even though 35 effective compounds of different acaricide groups such as pyrethroids or carbamates are available [2]. However, repeated or long-term chemical control may often lead to acaricide resistance of $D$. gallinae, as shown for carbaryl, permethrin, or DTT [5-8]. Increasing resistance combined with a lack of newly discovered acaricide substance groups show the importance of new development and intervention strategies to ensure animal welfare and to reduce economic losses in poultry farming. Such strategies might be targeted drug development by identifying drug targets and substance libraries screening. Alternatively, future control strategies could rely on vaccine development, which seems a feasible way to combat this hematophagous parasite. Homologous immunization of laying hens with soluble proteins extracted from D. gallinae achieved $50.6 \%$ mite mortality [9]. Heterologous immunization of poultry with recombinant Rhipicephalus microplus (formerly Boophilus microplus) Bm86, a membrane-bound midgut surface protein which is used as a vaccine antigen against the mentioned cattle tick, increased D. gallinae mortality by $23 \%$ (not significant) compared to the control group, whereas heterologous poultry immunization with recombinant subolesin originating from the mosquito Aedes albopictus increased D. gallinae mortality by $35.1 \%(\mathrm{p}=0.009)$ [10]. However, to date, no vaccine candidate with appropriate potential of mite control is available.

Excretory/secretory (ES) proteins play an important role in the host-parasite interface while acting as virulence factors or immune regulators to host immune recognition. Thus, they are crucial for survival of the parasite inside and outside the host organism [11,12]. As ES proteins are supposed to be involved in causing clinical infections in the host organism, they represent a favored group of antigens for the development of new therapeutical solutions e.g. as vaccine candidates or drug targets [12-14]. The current study was conducted to identify and functionally annotate putative ES (pES) and transmembrane (pTM) proteins of D. gallinae by in silico analysis of 454 pyrosequencing generated transcriptome data, which include all developmental stages of starved as well as fed mites [15]. These first analyses of the secretome as well as transmembranome of an acarid species provide potential D. gallinae drug targets or vaccine candidates against this major poultry pest.

\section{Methods}

\section{Identification of $D$. gallinae $\mathrm{pES}$ and $\mathrm{pTM}$ proteins}

D. gallinae $\mathrm{pES}$ and $\mathrm{pTM}$ protein identification was based on putative protein sequences of whole transcriptome data recently made available by Schicht et al. [15]. Those transcriptome data were generated by two 454pyrosequencing runs of a pooled cDNA sample of all developmental stages (from egg to the adult stage) and sex of starved as well as freshly blood fed D. gallinae mites. Conceptual translation of the resulting 267,464 D. gallinae nucleotide sequences produced 55,129 (20.6\%) coding regions derived from 17,860 isotigs, 24 contigs and 37,245 singletons.

In silico prediction of $\mathrm{pES}$ and $\mathrm{pTM}$ protein was carried out according to the protocol of Garg and Ranganathan [12], who conducted pES protein prediction by combining the computational tools SignalP [16], SecretomeP [17], TargetP $[18,19]$ and TMHMM $[20,21]$. The SignalP software package (version 4.1, http://www.cbs.dtu.dk/services/ SignalP/) was used for identifying classical secretory proteins. All putative D. gallinae proteins which were not classified to contain signal peptide cleavage sites were further analyzed with SecretomeP (version 2.0, http:// www.cbs.dtu.dk/services/SecretomeP/) for predicting nonclassical secreted proteins. To limit false positive results the neural network (NN) score of $\geq 0.9$ was set as described by Garg and Ranganathan [12]. To include only truly secreted D. gallinae proteins in subsequent analyses, proteins predicted to be secreted by either of the above mentioned software analyses were subsequently scanned for the presence of mitochondrial sequences by TargetP (version 1.1, http://www.cbs.dtu.dk/services/TargetP/) and transmembrane helices by TMHMM (version 2.0, http:// www.cbs.dtu.dk/services/TMHMM/). Protein sequences identified to be of mitochondrial origin or exhibiting transmembrane helices were excluded from the "secreted" data set. Prediction of D. gallinae pTM proteins was carried out separately by scanning the putative $D$. gallinae protein sequences with TMHMM.

\section{Identification of protein homologs}

For identifying homologous proteins, pES and pTM proteins were BLASTed (BLASTP) against the non-redundant 
(nr) database using the Blast2Go (b2g) software suite $[22,23]$. E-value cut-off was set at 1.0E-6.

\section{Functional annotation}

Supported by b2g, D. gallinae pES and pTM proteins were functionally mapped to Gene Ontology terms $[24,25]$ and annotated by setting default parameters (E-Value-Hit-Filter: 1.0E-6; Annotation cut-off: 55; GO weight: 5; Hsp-Hit Coverage cut-off: 0 ). Additionally, pES and pTM proteins were associated to protein families, domains and functional sites through InterProScan [26]. InterProScan integrated the following protein signature data bases: BlastProDom, FPrintScan, HMM-PIR, HMM-Pfam, HMM-Smart, HMM-Tigr, ProfileScan, Pattern Scan, Superfamily, Gene3D and HMM-Panther. pES and pTM proteins were subsequently passed to KOBAS2.0 [27] to identify statistically enriched related KO (KEGG Orthology) terms and KEGG pathways [28]. KOBAS was also used for KEGG gene mapping to identify pathways which are shared with the black-legged (deer) tick Ixodes scapularis as an example of a species belonging to the super order Parasitiformes. I. scapularis was chosen since its genome data are available [The Ixodes scapularis Genome project, http://extension.entm.purdue.edu/igp/; [29]].

\section{Results}

\section{Predicted D. gallinae secretome and transmembranome} size

Of the 55,129 putative D. gallinae protein sequences [15], a number of 2,935 sequences $(5.3 \%)$ were predicted to contain a signal peptide cleavage site by SignalP. Of the remaining sequences, SecretomeP classified 1,450 protein sequences $(2.6 \%)$ as non-classical secreted proteins. The putative 4,385 secreted proteins were parsed to TargetP and TMHMM resulting in 341 protein sequences (7.8\%) predicted to be of mitochondrial origin and 953 protein sequences $(21.7 \%)$ predicted to contain transmembrane helices. Potential mitochondrial and transmembrane proteins were excluded from the data set resulting in 3,091 D. gallinae pES protein sequences, representing $5.6 \%$ of the putative protein dataset. pTM protein prediction by TMHMM resulted in 7,361 (13.4\%) sequences out of the 55,129 D. gallinae putative protein sequences.

\section{pES and PTM protein identification}

Of the 3,091 D. gallinae pES protein sequences, a total of 1,083 (35.0\%) showed significant BLASTP matches with proteins deposited in the GenBank database. A percentage of $88.7 \%$ (961 sequences) of D. gallinae pES proteins matched with proteins of the phytoseiid predatory mite Metaseiulus occidentalis followed with a considerably lower number of sequences by the tick species I. scapularis and Rh. pulchellus (Figure 1). D. gallinae $\mathrm{pES}$ proteins were identified as proteases [e.g. cysteine proteases such as cathepsins (45 sequences) or legumains (11 sequences)], cuticle proteins (27 sequences), salivary proteins (24 sequences), mucins (5 sequences), vitellogenins (4 sequences) and many more. About $20 \%$ of pES proteins represented uncharacterized or hypothetical/predicted protein homologs. An overview of BLASTP top-hits is given in Table 1, whereas detailed BLASTP results are listed in Additional file 1.

BLASTP homology search of the 7,361 pTM protein sequences revealed 2,586 (35.1\%) matches with published protein sequences. Of these, 2,248 (86.9\%) were homologous to M. occidentalis (cf. Figure 1). Besides hypothetical and uncharacterized proteins, representing about $15 \%$ of sequence homologs, ion channels, receptors and several transporters [e.g. ABC transporter (12 sequences), glucose (12 sequences), amino acid (44 sequences), protein (12

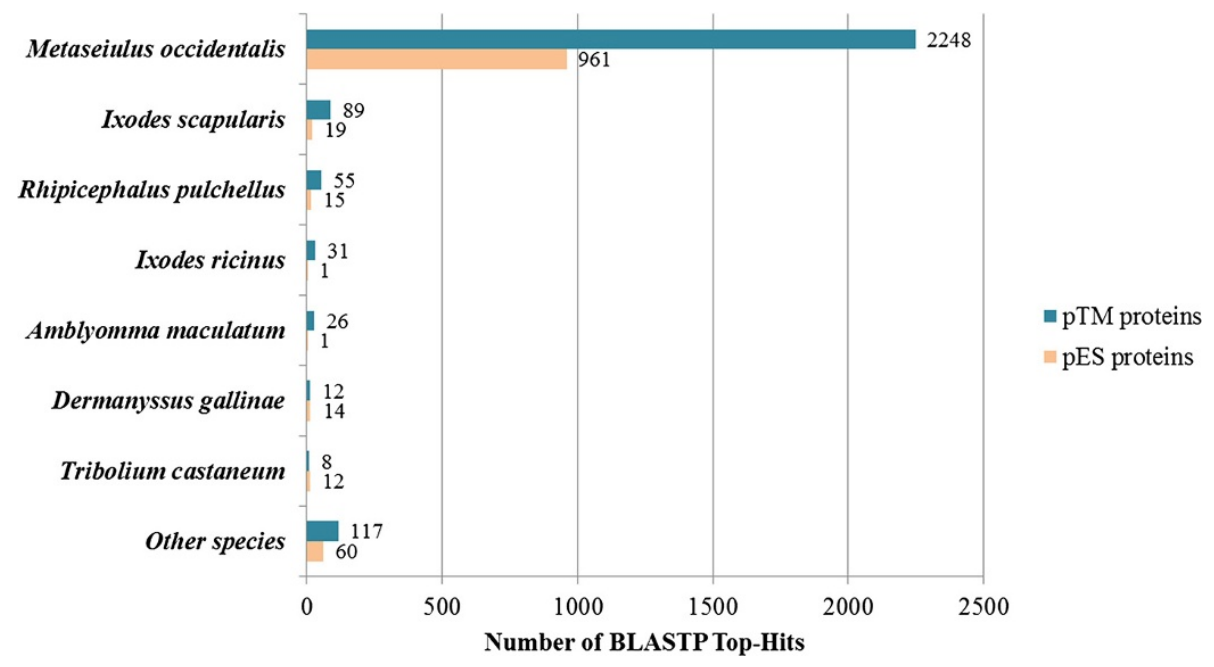

Figure 1 BLASTP top-hit species distribution of $D$. gallinae pES and pTM protein sequences. 
Table 1 Top 30 BLASTP matches of D. gallinae pES proteins

\begin{tabular}{|c|c|c|c|}
\hline Hit description & Species & Hit accession & No. of sequences \\
\hline Predicted: uncharacterized protein LOC100904214 & M. occidentalis & XP_003739349 & 15 \\
\hline Predicted: uncharacterized protein LOC100908413 & M. occidentalis & XP_003743523 & 11 \\
\hline Legumain-like protease precursor & M. occidentalis & XP_003746513 & 10 \\
\hline Cathepsin I-like & M. occidentalis & XP_003747496 & 10 \\
\hline Cathepsin I-1 & D. gallinae & CCC33064 & 9 \\
\hline TBC1 domain family member 10A-like & M. occidentalis & XP_003737545 & 8 \\
\hline Predicted: uncharacterized protein LOC100898836 & M. occidentalis & XP_003742669 & 8 \\
\hline Predicted: uncharacterized protein LOC100906884 & M. occidentalis & XP_003743278 & 8 \\
\hline Predicted: cathepsin L-like & M. occidentalis & XP_003743816 & 8 \\
\hline Predicted: cuticle protein 10.9-like & M. occidentalis & XP_003744493 & 8 \\
\hline Predicted: uncharacterized protein LOC100900702 & M. occidentalis & XP_003746592 & 8 \\
\hline Predicted: uncharacterized protein LOC100900705 & M. occidentalis & XP_003747300 & 8 \\
\hline Predicted: uncharacterized protein LOC100905853 & M. occidentalis & XP_003739512 & 7 \\
\hline Predicted: phospholipase A2-like & M. occidentalis & XP_003747592 & 7 \\
\hline Predicted: uncharacterized protein LOC100900008 & M. occidentalis & XP_003737797 & 6 \\
\hline Chymotrypsin-like elastase family member 2A-like & M. occidentalis & XP_003740761 & 6 \\
\hline Predicted: soluble calcium-activated nucleotidase 1-like & M. occidentalis & XP_003741943 & 6 \\
\hline Predicted: cathepsin L-like & M. occidentalis & XP_003738729 & 5 \\
\hline Predicted: peroxidasin-like & M. occidentalis & XP_003743011 & 5 \\
\hline Predicted: peroxiredoxin-4-like & M. occidentalis & XP_003743456 & 5 \\
\hline Predicted: probable serine carboxypeptidase CPVL-like isoform 1 & M. occidentalis & XP_003748333 & 5 \\
\hline Predicted: probable serine carboxypeptidase CPVL-like isoform 2 & M. occidentalis & XP_003748334 & 5 \\
\hline Secreted salivary gland & 1. persulcatus & BAH09304 & 4 \\
\hline Cathepsin D-1 & D. gallinae & CCC33063 & 4 \\
\hline Predicted: uncharacterized protein LOC100903770 & M. occidentalis & XP_003737317 & 4 \\
\hline Predicted: ferritin, liver middle subunit-like isoform 1 & M. occidentalis & XP_003737398 & 4 \\
\hline Predicted: cuticle protein 10.9-like & M. occidentalis & XP_003739044 & 4 \\
\hline Predicted: frizzled-4-like & M. occidentalis & XP_003740054 & 4 \\
\hline Predicted: epidermal growth factor receptor-like & M. occidentalis & XP_003740683 & 4 \\
\hline Predicted: uncharacterized protein LOC100905037 & M. occidentalis & XP_003740704 & 4 \\
\hline
\end{tabular}

sequences), zinc (24 sequences) or ion transporter (39 sequences)] could be identified. A large part of D. gallinae pTM protein sequences were assigned to ninjurin-like proteins (96 sequences). Furthermore, proteins of the mite's neuronal network such as GABA (2 sequences), glutamate (31 sequences) or acetylcholine (13 sequences) receptors and transporter (GABA: 11 sequences, glutamate: 4 sequences, acetylcholine: 2 sequences) were identified (Table 2 and Additional file 2).

\section{Functional annotation of $D$. gallinae pES and pTM proteins}

Functional annotation of D. gallinae pES and pTM proteins was based on Gene Ontology terms and assignment of protein families, protein domains and functional sites. Of the 3,091 D. gallinae pES proteins, 448 were assigned to $1,946 \mathrm{GO}$ terms divided into $870 \mathrm{GO}$ terms originating from the GO domain Biological Process, 383 GO terms from the Cellular Component domain and $693 \mathrm{GO}$ terms from the Molecular Function domain (Additional file 3). Of the latter, 574 GO terms could be assigned to a third level subcategory (Figure 2), whereby the term hydrolase activity represented with 140 annotations - e.g. cathepsin L, cathepsin K, legumain or secreted mucin - nearly one fourth of assigned GO terms. This term originates together with the terms isomerase activity ( 9 annotations), ligase activity (9), lyase activity (2), oxidoreductase activity (37), small proteins activating enzyme activity (1) and transferase activity (43) from the parental GO term catalytic activity, which included $42.0 \%$ of all Molecular Function Ontology terms assigned to a third level subcategory. The second largest share was contributed to by the parental 
Table 2 Top 30 BLASTP matches of D. gallinae pTM proteins

\begin{tabular}{|c|c|c|c|}
\hline Hit description & Species & Hit accession & No. of sequences \\
\hline Predicted: ninjurin-2-like & M. occidentalis & XP_003737707 & 96 \\
\hline Predicted: elongation of very long chain fatty acids protein 7-like & M. occidentalis & XP_003739733 & 50 \\
\hline Predicted: nose resistant to fluoxetine protein 6-like & M. occidentalis & XP_003740562 & 38 \\
\hline Hypothetical protein & Rh. pulchellus & JAA57419 & 28 \\
\hline Hypothetical protein & Rh. pulchellus & JAA54392 & 21 \\
\hline Predicted: steroid 17-alpha-hydroxylase/17,20 lyase-like & M. occidentalis & XP_003742972 & 18 \\
\hline Predicted: adipocyte plasma membrane-associated protein-like & M. occidentalis & XP_003748317 & 16 \\
\hline Predicted: cytochrome b561-like & M. occidentalis & XP_003737194 & 16 \\
\hline Predicted: voltage-dependent calcium channel type A subunit alpha-1-like & M. occidentalis & XP_003743405 & 14 \\
\hline Predicted: uncharacterized protein LOC100906895 & M. occidentalis & XP_003747421 & 13 \\
\hline Predicted: solute carrier family 22 member 6-like & M. occidentalis & XP_003744619 & 13 \\
\hline hypothetical protein & A. maculatum & AEO35694 & 12 \\
\hline Predicted: canalicular multispecific organic anion transporter 1, partial & M. occidentalis & XP_003738712 & 12 \\
\hline Predicted: uncharacterized protein LOC100907708 & M. occidentalis & XP_003742569 & 12 \\
\hline integral membrane protein, putative & 1. scapularis & XP_002412964 & 12 \\
\hline Predicted: excitatory amino acid transporter 4-like & M. occidentalis & XP_003737125 & 11 \\
\hline Predicted: uncharacterized protein LOC100904062 & M. occidentalis & XP_003737318 & 11 \\
\hline Predicted: lysophospholipid acyltransferase 7-like & M. occidentalis & XP_003746004 & 10 \\
\hline Predicted: PRA1 family protein 3-like & M. occidentalis & XP_003739732 & 10 \\
\hline Predicted: uncharacterized protein LOC100909236 & M. occidentalis & XP_003746970 & 10 \\
\hline Predicted: protein transport protein Sec61 subunit gamma-like & M. occidentalis & XP_003742336 & 10 \\
\hline Putative lipid exporter abca1, partial & Rh. pulchellus & JAA63751 & 9 \\
\hline Predicted: putative sodium-coupled neutral amino acid transporter 7-like & M. occidentalis & XP_003744784 & 9 \\
\hline Predicted: vacuolar ATPase assembly integral membrane protein VMA21-like & M. occidentalis & XP_003744117 & 9 \\
\hline Predicted: aquaporin-10-like & M. occidentalis & XP_003745025 & 8 \\
\hline Predicted: aldehyde dehydrogenase, dimeric NADP-preferring-like & M. occidentalis & XP_003746957 & 8 \\
\hline Putative caax prenyl protease 1 log danio rerio zinc metallopeptidase ste24 & 1. ricinus & JAA68353 & 8 \\
\hline Predicted: membrane-bound O-acyltransferase domain-containing protein 2-like & M. occidentalis & XP_003747942 & 8 \\
\hline Predicted: NADH dehydrogenase [ubiquinone] 1 beta subcomplex subunit 5, mitochondrial-like & M. occidentalis & XP_003738022 & 8 \\
\hline Predicted: patched domain-containing protein 3-like & M. occidentalis & XP_003747353 & 8 \\
\hline
\end{tabular}

term binding (34.2\%), represented amongst others by the third level subcategory terms protein binding (75 annotations), peptide binding (3), nucleic acid binding (34), carbohydrate binding (6) and lipid binding (3).

Of the total $7,361 \mathrm{D}$. gallinae pTM proteins, 1,253 were mapped to 6,610 GO terms originating from 3,097 Biological Processes terms, 1,720 Molecular Function terms and 1,793 Cellular Component terms (Additional file 4). On a third level subcategory, 1,637 GO terms were assigned to the Molecular Function domain (Figure 3), the largest part of which (325 annotations) being mapped to transmembrane transporter activity, followed by substrate-specific transporter activity (287) and hydrolase activity (196).

InterPro annotation of $\mathrm{pES}$ protein sequences resulted in 531 different assigned protein domains and families. A major part of D. gallinae pES proteins represented proteases assumed to be involved in proteolytic digestion. Another significant share was Immunoglobin-like proteins or subtypes involved in many functions such as cell-cell recognition, cell-surface receptors, muscle structure and immune functions. InterProScan of pTM protein sequences revealed 859 protein domains and families of which ninjurin was the most frequently occurring domain. A further large share of pTM protein sequences were assigned to different transporter systems such as ion channels, ABC transporter or symporter, and cytochrome P450 domains. The most frequently occurring protein domains and families of pES and pTM proteins of D. gallinae are shown in Table 3.

KEGG pathway mapping revealed $252 \mathrm{pES}$ proteins to be involved in 180 pathways. The most frequently assigned pathway was lysosome, followed by antigen processing 

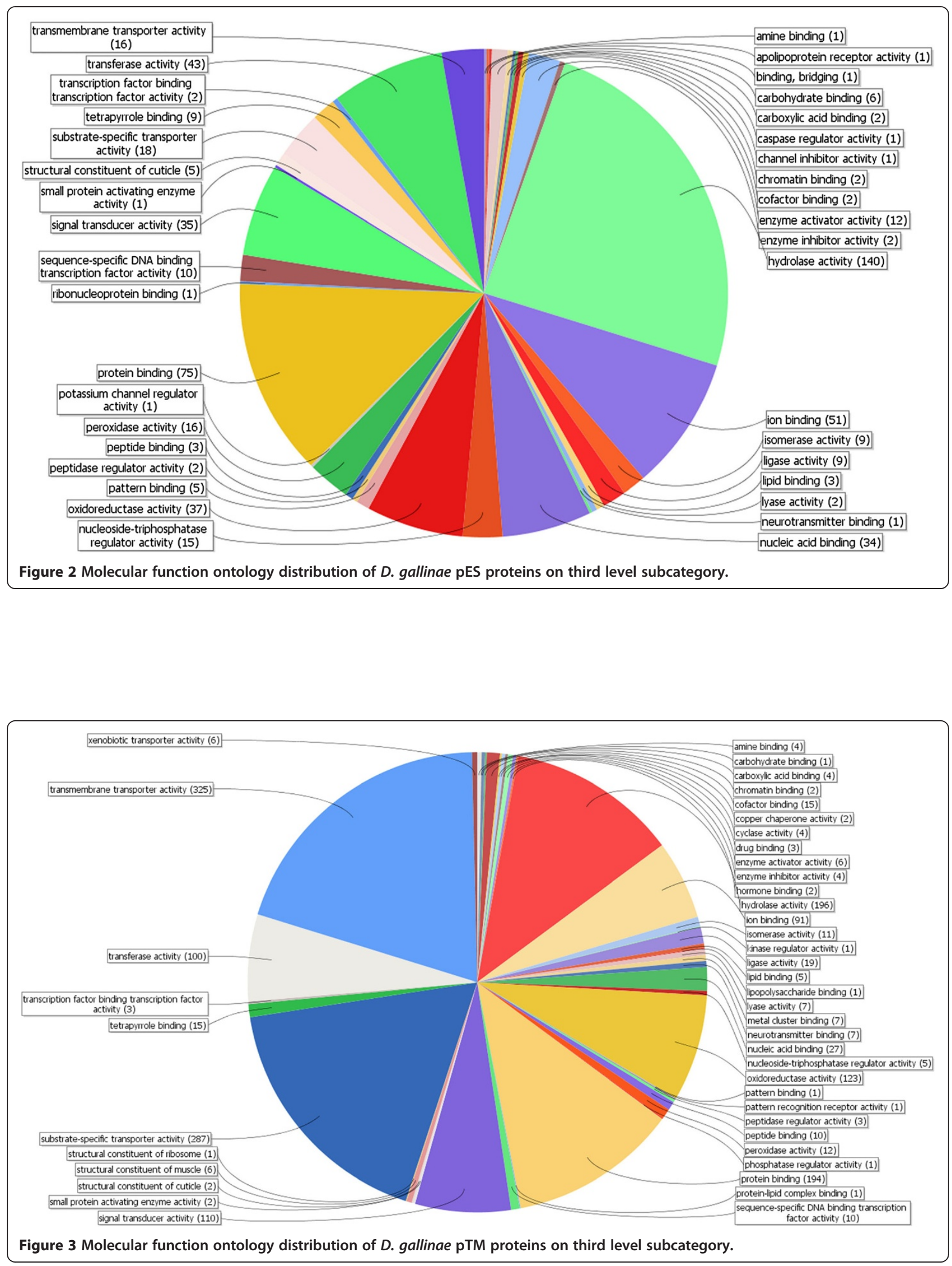
Table 3 Top 25 protein domains and families of $D$. gallinae pES and pTM proteins

\begin{tabular}{|c|c|c|c|}
\hline \multicolumn{2}{|l|}{ pES proteins } & \multicolumn{2}{|l|}{ pTM proteins } \\
\hline InterPro code and description & $\begin{array}{l}\text { No. of pES } \\
\text { proteins }(\%)\end{array}$ & InterPro code and description & $\begin{array}{l}\text { No. of pTM } \\
\text { proteins (\%) }\end{array}$ \\
\hline IPR013128 Peptidase C1A, papain & $42(1.36)$ & IPR007007 Ninjurin & $97(1.32)$ \\
\hline $\begin{array}{l}\text { IPR013201 Proteinase inhibitor 129, cathepsin } \\
\text { propeptide }\end{array}$ & $41(1.33)$ & $\begin{array}{l}\text { IPR016196 Major facilitator superfamily domain, general } \\
\text { substrate transporter }\end{array}$ & $56(0.76)$ \\
\hline IPR013783 Immunoglobulin-like fold & $34(1.10)$ & IPR002076 GNS1/SUR4 membrane protein & $53(0.72)$ \\
\hline $\begin{array}{l}\text { IPR009003 Peptidase cysteine/serine, trypsin- } \\
\text { like }\end{array}$ & $32(1.04)$ & IPR016040 NAD(P)-binding domain & $49(0.67)$ \\
\hline $\begin{array}{l}\text { IPR001254 Peptidase S1/S6, chymotrypsin/ } \\
\text { Hap }\end{array}$ & $31(1.00)$ & IPR001128 Cytochrome P450 & $46(0.62)$ \\
\hline IPR000618 Insect cuticle protein & $27(0.87)$ & IPR005821 Ion transport domain & $44(0.60)$ \\
\hline IPR007110 Immunoglobulin-like & $25(0.81)$ & IPR002401 Cytochrome P450, E-class, group I & $34(0.46)$ \\
\hline IPR000668 Peptidase C1A, papain C-terminal & $24(0.78)$ & IPR017452 GPCR, rhodopsin-like, 7TM & $30(0.41)$ \\
\hline $\begin{array}{l}\text { IPR001314 Peptidase S1A, chymotrypsin- } \\
\text { type }\end{array}$ & $24(0.78)$ & IPR000175 Sodium:neurotransmitter symporter & $29(0.39)$ \\
\hline $\begin{array}{l}\text { IPR018114 Peptidase S1/S6, chymotrypsin/ } \\
\text { Hap, active site }\end{array}$ & $23(0.74)$ & IPR002198 Short-chain dehydrogenase/reductase SDR & $29(0.39)$ \\
\hline $\begin{array}{l}\text { IPR000169 Cysteine peptidase, cysteine } \\
\text { active site }\end{array}$ & $22(0.71)$ & IPR013783 Immunoglobulin-like fold & $29(0.39)$ \\
\hline IPR012336 Thioredoxin-like fold & $18(0.58)$ & IPR000276 GPCR, rhodopsin-like & $27(0.37)$ \\
\hline $\begin{array}{l}\text { IPR025661 Cysteine peptidase, asparagine } \\
\text { active site }\end{array}$ & $18(0.58)$ & IPR002347 Glucose/ribitol dehydrogenase & $27(0.37)$ \\
\hline IPR013098 Immunoglobulin I-set & $14(0.45)$ & IPR001140 ABC transporter, transmembrane domain & $24(0.33)$ \\
\hline $\begin{array}{l}\text { IPR013781 Glycoside hydrolase, catalytic } \\
\text { domain }\end{array}$ & $14(0.45)$ & IPR017940 ABC transporter, integral membrane type 1 & $24(0.33)$ \\
\hline IPR017853 Glycoside hydrolase, superfamily & $14(0.45)$ & IPR020846 Major facilitator superfamily domain & $24(0.33)$ \\
\hline IPR002007 Heme peroxidase, animal & $13(0.42)$ & IPR017972 Cytochrome P450, conserved site & $23(0.31)$ \\
\hline IPR010255 Heme peroxidase & $13(0.42)$ & IPR011527 ABC transporter, transmembrane domain, type 1 & $22(0.30)$ \\
\hline IPR003598 Immunoglobulin subtype 2 & $12(0.39)$ & IPR011042 Six-bladed beta-propeller, TolB-like & $21(0.29)$ \\
\hline IPR003599 Immunoglobulin subtype & $12(0.39)$ & IPR013099 Ion transport 2 & $21(0.29)$ \\
\hline IPR001096 Peptidase C13, legumain & $11(0.36)$ & IPR006201 Neurotransmitter-gated ion-channel & $20(0.27)$ \\
\hline $\begin{array}{l}\text { IPR001563 Peptidase S10, serine } \\
\text { carboxypeptidase }\end{array}$ & $11(0.36)$ & IPR004299 Membrane bound O-acyl transferase, MBOAT & $19(0.26)$ \\
\hline IPR013083 Zinc finger, RING/FYVE/PHD-type & $11(0.36)$ & IPR011701 Major facilitator superfamily & $18(0.24)$ \\
\hline IPR002018 Carboxylesterase, type B & $10(0.32)$ & IPR005828 General substrate transporter & $17(0.23)$ \\
\hline IPR011009 Protein kinase-like domain & $10(0.32)$ & IPR018108 Mitochondrial substrate/solute carrier & $17(0.23)$ \\
\hline
\end{tabular}

and presentation. Phagosome was the third most frequent pathway. Mapping of $D$. gallinae pES protein sequences against $I$. scapularis KEGG GENES resulted in assigning $133 \mathrm{pES}$ proteins to 60 pathways. The analysis revealed lysosome as the most frequent and phagosome as the third most frequent pathway as well, whereas the second most frequent pathway was represented by metabolic pathways. The top 15 KEGG pathways as well as I. scapularis pathways of pES protein sequences are listed in Table 4. A total of 611 pTM protein sequences were found to be involved in 210 KEGG pathways with protein processing in endoplasmic reticulum being the most frequently occurring pathway, followed by lysosome and Huntington's disease (cf. Table 5). Furthermore, pTM protein sequences were mapped to neuronal processes such as neuroactive ligand-receptor interaction or glutamatergic synapse. KEGG Gene mapping of D. gallinae pTM protein sequences to $I$. scapularis revealed 331 pTM protein sequences to be involved in 60 pathways. The most frequently shared assigned pathway were metabolic pathways followed by protein processing in endoplasmic reticulum and lysosome.

\section{Discussion}

The secretome is a part of the proteome of an organism and includes all proteins secreted by the cell including 
Table 4 Top 15 KEGG pathways of $D$. gallinae pES proteins

\begin{tabular}{|c|c|c|c|c|c|}
\hline KEGG pathway & $\begin{array}{l}\text { No. of } \\
\text { sequences (\%) }\end{array}$ & $\begin{array}{l}\text { No. of } \\
\text { enzymes }\end{array}$ & I. sapularis KEGG pathway & $\begin{array}{l}\text { No. of } \\
\text { sequences (\%) }\end{array}$ & $\begin{array}{l}\text { No. of NCBI } \\
\text { Gene ID's }\end{array}$ \\
\hline ko04142 Lysosome & $58(1.88)$ & 17 & isc04142 Lysosome & $41(1.33)$ & 12 \\
\hline $\begin{array}{l}\text { ko04612 Antigen processing and } \\
\text { presentation }\end{array}$ & $37(1.20)$ & 5 & isc01100 Metabolic pathways & $26(0.84)$ & 21 \\
\hline ko04145 Phagosome & $29(0.94)$ & 7 & isc04145 Phagosome & $18(0.58)$ & 6 \\
\hline ko05323 Rheumatoid arthritis & $22(0.71)$ & 4 & $\begin{array}{l}\text { isc04141 Protein processing in } \\
\text { endoplasmic reticulum }\end{array}$ & $17(0.55)$ & 12 \\
\hline $\begin{array}{l}\text { ko04141 Protein processing in } \\
\text { endoplasmic reticulum }\end{array}$ & $19(0.61)$ & 14 & isc04310 Wnt signaling pathway & $9(0.2)$ & 6 \\
\hline ko00230 Purine metabolism & $16(0.52)$ & 7 & isc00230 Purine metabolism & $8(0.26)$ & 5 \\
\hline ko00240 Pyrimidine metabolism & $16(0.52)$ & 7 & isc00240 Pyrimidine metabolism & $8(0.26)$ & 5 \\
\hline ko05166 HTLV-I infection & $14(0.45)$ & 8 & isc00511 Other glycan degradation & $6(0.19)$ & 3 \\
\hline ko04310 Wnt signaling pathway & $12(0.39)$ & 7 & $\begin{array}{l}\text { isc03008 Ribosome biogenesis in } \\
\text { eukaryotes }\end{array}$ & $6(0.19)$ & 3 \\
\hline ko05200 Pathways in cancer & $12(0.39)$ & 6 & isc03018 RNA degradation & $5(0.16)$ & 2 \\
\hline ko04510 Focal adhesion & $11(0.36)$ & 7 & $\begin{array}{l}\text { isc } 04120 \text { Ubiquitin mediated } \\
\text { proteolysis }\end{array}$ & $4(0.13)$ & 4 \\
\hline $\begin{array}{l}\text { ko00860 Porphyrin and chlorophyll } \\
\text { metabolism }\end{array}$ & $9(0.29)$ & 3 & isc00600 Sphingolipid metabolism & $4(0.13)$ & 3 \\
\hline ko04010 MAPK signaling pathway & $9(0.29)$ & 6 & isc03013 RNA transport & $4(0.13)$ & 3 \\
\hline ko04972 Pancreatic secretion & $9(0.29)$ & 7 & $\begin{array}{l}\text { isc04070 Phosphatidylinositol signaling } \\
\text { system }\end{array}$ & $4(0.13)$ & 3 \\
\hline ko04020 Calcium signaling pathway & $8(0.26)$ & 5 & isc03015 mRNA surveillance pathway & $4(0.13)$ & 2 \\
\hline
\end{tabular}

Table 5 Top 15 KEGG pathways of $D$. gallinae pTM proteins

\begin{tabular}{|c|c|c|c|c|c|}
\hline KEGG pathway & $\begin{array}{l}\text { No. of } \\
\text { sequences (\%) }\end{array}$ & $\begin{array}{l}\text { No. of } \\
\text { enzymes }\end{array}$ & I. sapularis KEGG pathway & $\begin{array}{l}\text { No. of } \\
\text { sequences (\%) }\end{array}$ & $\begin{array}{l}\text { No of NCBI } \\
\text { Gene ID's }\end{array}$ \\
\hline $\begin{array}{l}\text { ko04141 Protein processing in } \\
\text { endoplasmic reticulum }\end{array}$ & $60(0.81)$ & 25 & isc01100 Metabolic pathways & $104(1.43)$ & 52 \\
\hline ko04142 Lysosome & $52(0.71)$ & 15 & $\begin{array}{l}\text { isc } 04141 \text { Protein processing in } \\
\text { endoplasmic reticulum }\end{array}$ & $54(0.73)$ & 21 \\
\hline ko05016 Huntington's disease & $52(0.71)$ & 21 & isc04142 Lysosome & $50(0.68)$ & 13 \\
\hline ko00190 Oxidative phosphorylation & $48(0.65)$ & 24 & isc00190 Oxidative phosphorylation & $39(0.53)$ & 16 \\
\hline ko04020 Calcium signaling pathway & $44(0.6)$ & 18 & isc04145 Phagosome & $23(0.31)$ & 8 \\
\hline ko05010 Alzheimer's disease & $43(0.58)$ & 21 & $\begin{array}{l}\text { isc } 04080 \text { Neuroactive ligand-receptor } \\
\text { interaction }\end{array}$ & $22(0.30)$ & 11 \\
\hline $\begin{array}{l}\text { ko04080 Neuroactive ligand-receptor } \\
\text { interaction }\end{array}$ & $42(0.57)$ & 24 & isc03060 Protein export & $20(0.27)$ & 7 \\
\hline ko04724 Glutamatergic synapse & $42(0.57)$ & 16 & $\begin{array}{l}\text { isc00564 Glycerophospholipid } \\
\text { metabolism }\end{array}$ & $19(0.26)$ & 7 \\
\hline ko05012 Parkinson's disease & $41(0.56)$ & 16 & isc00510 N-Glycan biosynthesis & $18(0.24)$ & 10 \\
\hline ko02010 ABC transporters & $34(0.46)$ & 15 & isc04146 Peroxisome & $16(0.22)$ & 6 \\
\hline ko04972 Pancreatic secretion & $34(0.46)$ & 14 & isc04310 Wnt signaling pathway & $13(0.18)$ & 6 \\
\hline $\begin{array}{l}\text { ko00564 Glycerophospholipid } \\
\text { metabolism }\end{array}$ & $32(0.43)$ & 13 & isc00600 Sphingolipid metabolism & $11(0.15)$ & 6 \\
\hline ko04970 Salivary secretion & $30(0.41)$ & 13 & $\begin{array}{l}\text { isc04120 Ubiquitin mediated } \\
\text { proteolysis }\end{array}$ & $11(0.15)$ & 6 \\
\hline ko04145 Phagosome & $27(0.37)$ & 12 & $\begin{array}{l}\text { isc } 01040 \text { Biosynthesis of unsaturated } \\
\text { fatty acids }\end{array}$ & $10(0.14)$ & 4 \\
\hline ko04723 Retrograde endocannabinoid & $28(0.38)$ & 14 & isc00020 Citrate cycle (TCA cycle) & $9(0.12)$ & 4 \\
\hline
\end{tabular}


those of the extracellular matrix, proteins shed from the cell membrane and vesicle proteins like microsomal vesicles [12,30-32]. Transmembrane proteins are a group of membrane proteins containing subunits exposed on both sides of a cell membrane. They compose approximately $30 \%$ of a typical genome and are involved in many important biological processes including cell signaling, transport of membrane-impermeable molecules and cell recognition [33]. As the present D. gallinae secretome as well as transmembranome predictions and analyses were based on putative proteins of transcriptome data including all developmental stages of starved as well as fed whole body poultry red mites [15], a broad spectrum of pES and pTM proteins originating from different metabolic pathways was expected. About one fifth (19\%) of D. gallinae putative proteins was assigned to the mite's secretome or transmembranome, divided into 5.6\% $(3,091)$ secreted and $13.4 \%(7,361)$ transmembrane proteins, respectively. Of these predicted proteins, $~ 35.0 \%$ showed significant matches with known protein sequences, with the predatory phytoseiid mite $M$. occidentalis being the top-1 hit of $89 \%$ D. gallinae pES proteins and $87 \%$ pTM proteins. This was not unexpected since both mite species share a close relationship and furthermore, the genome as well as the transcriptome of M. occidentalis is sequenced and available in the common data bases [34,35]. The share of $65 \% \mathrm{pES}$ and pTM proteins which could not be identified via BLAST and thus were categorized as "novel" are either parasite specific proteins or even unique for the poultry red mite, underlining the importance of further research on protein characterization.

The major part of pES proteins were identified as hydrolases and of these, a large share was cysteine proteases. Cysteine proteases are important in different biochemical and physiological processes of arthropods like embryogenesis [36-42]. The arthropod embryo needs a lot of nutrients during its development. It obtains its nutrition from egg reserve material consisting of amino acids, carbohydrates and lipids stored in yolk granules. To make these nutrients available, enzymatic machinery is needed [39]. Degradation of the yolk protein vitellin is triggered by acidification of the yolk granules, activating as a consequence cysteine proteinases like cathepsin $\mathrm{L}$ and $\mathrm{B}$ $[37-39,43]$ and aspartic proteinases like cathepsin D [44]. Besides embryogenesis, these cathepsins may be essential in the proteolytic digestion of the mite's blood meal. This might be extrapolated from the well-studied blood digestion in the closely related ticks [45-49] since both, ticks and D. gallinae share anatomic similarity of the intestinal tract belonging to the Parasitiformes type. As summarized by Horn et al. [45], the primary cleavage of hemoglobin in the hard tick I. ricinus is accomplished by the endopeptidase cathepsin D, which is supported by the catalytic activity of cathepsin L and legumain. These three proteases represent 6 of the top-30 BLASTP matches of the poultry red mite's secretome (cf. Table 1 ). The protein family and domain analysis of $D$. gallinae $\mathrm{pES}$ proteins revealed a high proportion of cysteine peptidases $\mathrm{C} 1 \mathrm{~A}$, papain (IPR013128: 42 pES sequences; IPR000668: 22 pES sequences) and C13, legumain (IPR001096: 11 pES sequences). Santamaria et al. [50] compared the number of different protein families of the phytophageous mite Tetranychus urticae to different arthropod species (genome data of 10 insects, 1 crustacean and 1 tick) and found that C1A papain-like peptidases are common in all species, whereas a high number of $\mathrm{C} 13$ legumain-like peptidases (19 genes) was found in T. urticae mites compared to the other arthropods. The eleven predicted legumainlike peptidases in D. gallinae suggest that extensive expansion of this protein family amongst mites might not be unusual. KEGG pathway mapping predicted pES proteins to be most frequently located in the lysosome in both pathways of all organisms and the tick I. scapularis alone. This might result from the large number of $D$. gallinae pES proteins identified as proteases like cathepsins, which may act as endopeptidases in the lysosome [51]. As in mites, digestive processes of ticks take place in the acid endosomal/ lysosomal vesicles of gut epithelia cells, contrary to other blood feeding arthropods [52,53].

With the identification and analyses of different blood feeding-induced molecules of ticks, an antigen against the cattle tick Rhipicephalus microplus (formerly Boophilus microplus) was found [54] leading to successful vaccine development against this tick (TickGARD plus $^{\mathrm{Tm}} / \mathrm{Gavac}^{\mathrm{Tw}}$ ). The antigen Bm86 is a membrane-bound glycoprotein of the tick's intestinal tract $[54,55]$. As a gut protein, Bm86 is not part of the normal host-tick interaction and therefore does not stimulate an immunological response under normal circumstances. However, vaccination with this "concealed" or "hidden" antigen induced an immunological response of the cattle host consequently damaging the blood feeding tick $[56,57]$. When considering potential feeding-induced predicted D. gallinae pTM and pES molecules as concealed antigens, a broad range of new vaccine candidates against the poultry red mite is provided by the present study, e.g. legumains, chymotrypsins or cathepsins. Of these, cathepsin D and L were suggested by Bartley et al. [58] as part of a multi-component vaccine against the poultry red mite due to their efficiency in an in vitro feeding assay. Notably, BLASTP search of predicted D. gallinae pTM proteins did not result in a Rh. microplus Bm86 hit amongst the top-20 BLAST Hits of each D. gallinae PTM protein sequence. This may indicate that no Bm86 homologue is present in the poultry red mite, although as this search was based on transcriptomic data it could be present albeit at low abundance or alternatively it may not be expressed. Specific analysis of the mite's gut transcripts could test this hypothesis. However, in contrast 
to ticks, dissection of D. gallinae's gut is not possible [59] due to its small size. This is, depending on the developmental stage, about $0.39-1 \mathrm{~mm}$ in length and 0.26$0.64 \mathrm{~mm}$ in width [60]. Thus, estimating gut-derived pTM proteins remains complicated as proteases and other molecules suggested to be involved in blood digestion may also play a role in other processes. Besides "hidden" antigens "exposed" antigens like salivary proteins are also discussed as vaccine candidates against ticks $[57,61]$ as they may act as immunomodulators $[62,63]$. Therefore, further research on the 24 predicted salivary proteins of $D$. gallinae could also promote vaccine development.

The BLASTP top-hit of the D. gallinae transmembranome is represented by a ninjurin-2-like protein. In mammals, ninjurin acts as an adhesion molecule which is induced by nerve injury and promotes axonal growth but is also expressed in a number of other tissues, predominantly in epithelial cells [64]. Correlation of ninjurin upregulation with wounding incidents was also shown in adult Drosophila melanogaster flies [65] and in the mosquito Anopheles gambiae ninjurin is suggested to play an important role in innate immune response, probably in signaling and cell communication [66]. Whether the abundance of ninjurin-like proteins amongst the D. gallinae PTM proteins results from the preparation steps (immobilized living mites were collected via forceps which might have injured the mites), from immune functions or from completely different, hitherto unknown functions in mites remains unclear. Another interesting and unexpected pTM protein homolog was the third most common BLASTP top-hit, which is predicted as being nose resistant to fluoxetine protein 6-like (nrf-6). This transmembrane protein, which acts in the intestine of Caenorhabditis elegans and confers sensitivity to the antidepressant fluoxetine (Prozak), is suggested to play a role in drug or yolk transport across membranes $[67,68]$. The D. melanogaster beltless (blt) gene shares homologies with the C. elegans nrf- 6 and is crucial during oogenesis and embryogenesis, but also expressed in the adult nervous system and thus suggested to be important for neuronal functioning [69]. In mites, function and localization of genes involved in neurological processes is largely unknown. Research work is needed to characterize D. gallinae pTM proteins involved in neurological processes, since - if one extrapolates from the mode of action of most acaricides - they could represent promising candidates for identifying new drug targets against poultry red mites.

\section{Conclusion}

The present study is the first of its kind analyzing an acarid secretome as well as transmembranome in silico. It provides valuable insights into a pool of $D$. gallinae proteins, which might be relatively easily accessible candidates for drugs or immunological components like antibodies and thus represent potential drug targets or vaccine candidates. In particular, pES or pTM proteins suggested to be involved in blood feeding and digestion or neurological processes provide a promising basis for further research on new intervention strategies against $D$. gallinae, which is considered one of the most serious pests of poultry. Nevertheless, future studies on the proteomic level are highly desirable to confirm the predicted secretome and transmembranome of the red poultry mite as in silico analyses rely on the use of algorithms to identify sequence features (i.e. signal peptide motifs) and thus do not necessarily accurately reflect the entire biological truth.

\section{Additional files}

Additional file 1: BLASTP top-hits of $D$. gallinae $\mathrm{pES}$ proteins.

Additional file 2: BLASTP top-hits of $D$. gallinae pTM proteins.

Additional file 3: Gene Ontology term distribution of $D$. gallinae pES proteins.

Additional file 4: Gene Ontology term distribution of $D$. gallinae pTM proteins.

\section{Competing interests}

The authors declare that they have no competing interests.

\section{Authors' contributions}

CS conceived and designed the research plan, coordinated the study and participated in writing of the manuscript. SS and WQ performed bioinformatic analyses. SS, WQ, LP and CS analyzed and interpreted the data. SS drafted the manuscript. All authors read and approved the final manuscript.

\section{Author details}

'Institute for Parasitology, University of Veterinary Medicine Hannover, Buenteweg 17, 30559 Hannover, Germany. ${ }^{2}$ Functional Genomics Center Zurich, Winterthurerstrasse 190, 8057 Zurich, Switzerland.

Received: 20 August 2013 Accepted: 8 September 2013

Published: 11 September 2013

\section{References}

1. Kirkwood AC: Anaemia in poultry infested with the red mite Dermanyssus gallinae. Vet Rec 1967, 80:514-516.

2. Chauve C: The poultry red mite Dermanyssus gallinae (De Geer, 1778): current situation and future prospects for control. Vet Parasitol 1998, 79:239-245.

3. Sparagano O, Pavlicevic A, Murano T, Camarda A, Sahibi H, Kilpinen O, Mul M, van Emous R, le Bouquin S, Hoel K, Cafiero MA: Prevalence and key figures for the poultry red mite Dermanyssus gallinae infections in poultry farm systems. Exp Appl Acarol 2009, 48:3-10.

4. Hoglund J, Nordenfors H, Uggla A: Prevalence of the poultry red mite, Dermanyssus gallinae, in different types of production systems for egg layers in Sweden. Poult Sci 1995, 74:1793-1798.

5. Beugnet F, Chauve C, Gauthey M, Beert L: Resistance of the red poultry mite to pyrethroids in France. Vet Rec 1997, 140:577-579.

6. Marangi M, Cafiero MA, Capelli G, Camarda A, Sparagano OA, Giangaspero A: Evaluation of the poultry red mite, Dermanyssus gallinae (Acari: Dermanyssidae) susceptibility to some acaricides in field populations from Italy. Exp Appl Acarol 2009, 48:11-18.

7. Zeman P, Zelezny J: The susceptibility of the poultry red mite, Dermanyssus gallinae (De Geer, 1778), to some acaricides under laboratory conditions. Exp Appl Acarol 1985, 1:17-22. 
8. Zeman P: Encounter the poultry red mite resistance to acaricides in Czechoslovak poultry-farming. Folia Parasitol (Praha) 1987, 34:369-373.

9. Harrington D, Din HM, Guy J, Robinson K, Sparagano O: Characterization of the immune response of domestic fowl following immunization with proteins extracted from Dermanyssus gallinae. Vet Parasitol 2009, 160:285-294.

10. Harrington D, Canales M, de la Fuente J, de Luna C, Robinson K, Guy J, Sparagano O: Immunisation with recombinant proteins subolesin and Bm86 for the control of Dermanyssus gallinae in poultry. Vaccine 2009, 27:4056-4063

11. Hewitson JP, Grainger JR, Maizels RM: Helminth immunoregulation: the role of parasite secreted proteins in modulating host immunity. Mol Biochem Parasitol 2009, 167:1-11.

12. Garg G, Ranganathan S: In silico secretome analysis approach for next generation sequencing transcriptomic data. BMC Genomics 2011, 12(Suppl 3):S14

13. Hotez PJ, Zhan B, Bethony JM, Loukas A, Williamson A, Goud GN, Hawdon JM, Dobardzic A, Dobardzic R, Ghosh K, et al: Progress in the development of a recombinant vaccine for human hookworm disease: the human hookworm vaccine initiative. Int J Parasitol 2003, 33:1245-1258.

14. Bonin-Debs AL, Boche I, Gille H, Brinkmann U: Development of secreted proteins as bio therapeutic agents. Expert Opin Biol Ther 2004, 4:551-558.

15. Schicht S, Qi W, Poveda L, Strube C: Whole transcriptome analysis of the poultry red mite Dermanyssus gallinae (De Geer, 1778). Parasitology. in press.

16. Petersen TN, Brunak S, von Heijne G, Nielsen H: SignalP 4.0: discriminating signal peptides from transmembrane regions. Nat Methods 2011, 8:785-786.

17. Bendtsen JD, Jensen LJ, Blom N, Von Heijne G, Brunak S: Feature-based prediction of non-classical and leaderless protein secretion. Protein Eng Des Sel 2004, 17:349-356.

18. Emanuelsson $\mathrm{O}$, Nielsen $\mathrm{H}$, Brunak $\mathrm{S}$, von Heijne G: Predicting subcellular localization of proteins based on their $\mathrm{N}$-terminal amino acid sequence J Mol Biol 2000, 300:1005-1016.

19. Emanuelsson O, Brunak S, von Heijne G, Nielsen H: Locating proteins in the cell using TargetP, SignalP and related tools. Nat Protoc 2007, 2:953-971.

20. Sonnhammer EL, von Heijne G, Krogh A: A hidden Markov model for predicting transmembrane helices in protein sequences. Proc Int Conf Intell Syst Mol Biol 1998, 6:175-182.

21. Krogh A, Larsson B, von Heijne G, Sonnhammer EL: Predicting transmembrane protein topology with a hidden Markov model: application to complete genomes. J Mol Biol 2001, 305:567-580.

22. Conesa A, Gotz S, Garcia-Gomez JM, Terol J, Talon M, Robles M: Blast2GO: a universal tool for annotation, visualization and analysis in functional genomics research. Bioinformatics 2005, 21:3674-3676.

23. Gotz S, Garcia-Gomez JM, Terol J, Williams TD, Nagaraj SH, Nueda MJ, Robles M, Talon M, Dopazo J, Conesa A: High-throughput functional annotation and data mining with the Blast2GO suite. Nucleic Acids Res 2008, 36:3420-3435.

24. Ashburner M, Ball CA, Blake JA, Botstein D, Butler H, Cherry JM, Davis AP, Dolinski K, Dwight SS, Eppig JT, et al: Gene ontology: tool for the unification of biology. The gene ontology consortium. Nat Genet 2000, 25:25-29.

25. The Gene Ontology Consortium: The gene ontology project in 2008. Nucleic Acids Res 2008, 36:D440-D444.

26. Hunter $S$, Jones $P$, Mitchell A, Apweiler R, Attwood TK, Bateman A, Bernard T, Binns D, Bork P, Burge S, et al: InterPro in 2011: new developments in the family and domain prediction database. Nucleic Acids Res 2011, 40:D306-D312.

27. Xie C, Mao X, Huang J, Ding Y, Wu J, Dong S, Kong L, Gao G, Li CY, Wei L: KOBAS 2.0: a web server for annotation and identification of enriched pathways and diseases. Nucleic Acids Res 2011, 39:W316-W322.

28. Kanehisa M, Araki M, Goto S, Hattori M, Hirakawa M, Itoh M, Katayama T, Kawashima S, Okuda S, Tokimatsu T, Yamanishi Y: KEGG for linking genomes to life and the environment. Nucleic Acids Res 2008 36:D480-D484.

29. Hill CA, Wikel SK: The Ixodes scapularis genome project: an opportunity for advancing tick research. Trends Parasitol 2005, 21:151-153.

30. Caccia D, Dugo M, Callari M, Ongarzone I: Bioinformatics tools for secretome analysis. Biochim Biophys Acta 2013. in press.

31. Antelmann $H$, Tjalsma H, Voigt B, Ohlmeier S, Bron S, van Dijl JM, Hecker M A proteomic view on genome-based signal peptide predictions. Genome Res 2001, 11:1484-1502.
32. Tjalsma H, Bolhuis A, Jongbloed JD, Bron S, van Dijl JM: Signal peptide-dependent protein transport in Bacillus subtilis: a genome-based survey of the secretome. Microbiol Mol Biol Rev 2000, 64:515-547.

33. Nugent T, Jones DT: Transmembrane protein topology prediction using support vector machines. BMC Bioinforma 2009, 10:159.

34. Hoy MA: The predatory mite Metaseiulus occidentalis: mitey small and mitey large genomes. Bioessays 2009, 31:581-590.

35. Hoy MA, Yu F, Meyer JM, Tarazona OA, Jeyaprakash A, Wu K: Transcriptome sequencing and annotation of the predatory mite Metaseiulus occidentalis (Acari: Phytoseiidae): a cautionary tale about possible contamination by prey sequences. Exp Appl Acarol 2012, 59:283-296.

36. Sojka D, Francischetti IM, Calvo E, Kotsyfakis M: Cysteine proteases from blood feeding arthropod ectoparasites. Adv Exp Med Biol 2011, 712:177-191.

37. Fagotto F: Yolk degradation in tick eggs: II. Evidence that cathepsin L-like proteinase is stored as a latent, acid-activable proenzyme. Arch Insect Biochem Physiol 1990, 14:237-252.

38. Fagotto F: Yolk degradation in tick eggs: I. Occurrence of a cathepsin L-like acid proteinase in yolk spheres. Arch Insect Biochem Physiol 1990, 14:217-235

39. Seixas A, Dos Santos PC, Velloso FF, Da Silva Vaz I Jr, Masuda A, Horn F, Termignoni C: A Boophilus microplus vitellin-degrading cysteine endopeptidase. Parasitology 2003, 126:155-163.

40. Estrela A, Seixas A, Termignoni C: A cysteine endopeptidase from tick (Rhipicephalus (Boophilus) microplus) larvae with vitellin digestion activity. Comp Biochem Physiol B Biochem Mol Biol 2007, 148:410-416.

41. Uchida K, Ohmori D, Ueno T, Nishizuka M, Eshita Y, Fukunaga A, Kominami E: Preoviposition activation of Cathepsin-like proteinases in degenerating ovarian follicles of the mosquito Culex pipiens pallens. Dev Biol 2001, 237:68-78.

42. Cho WL, Tsao SM, Hays AR, Walter R, Chen JS, Snigirevskaya ES, Raikhel AS: Mosquito Cathepsin B-like protease involved in embryonic degradation of vitellin is produced as a latent extra ovarian precursor. J Biol Chem 1999, 274:13311-13321.

43. Medina M, Leon P, Vallejo CG: Drosophila Cathepsin B-like proteinase: a suggested role in yolk degradation. Arch Biochem Biophys 1988, 263:355-363.

44. Logullo C, Vaz Ida S, Sorgine MH, Paiva-Silva GO, Faria FS, Zingali RB, De Lima MF, Abreu L, Oliveira EF, Alves EW, et al: Isolation of an aspartic proteinase precursor from the egg of a hard tick, Boophilus microplus. Parasitology 1998, 116(Pt 6):525-532.

45. Horn M, Nussbaumerova M, Sanda M, Kovarova Z, Srba J, Franta Z, Sojka D, Bogyo M, Caffrey CR, Kopacek P, Mares M: Hemoglobin digestion in blood-feeding ticks: mapping a multipeptidase pathway by functional proteomics. Chem Biol 2009, 16:1053-1063.

46. Ribeiro JM, Labruna MB, Mans BJ, Maruyama SR, Francischetti IM, Barizon GC, dMS IK: The sialotranscriptome of Antricola delacruzi female ticks is compatible with non-hematophagous behavior and an alternative source of food. Insect Biochem Mol Biol 2012, 42:332-342.

47. Alim MA, Tsuji N, Miyoshi T, Islam MK, Hatta T, Yamaji K, Fujisaki K: Developmental stage- and organ-specific expression profiles of asparaginyl endopeptidases/legumains in the ixodid tick Haemaphysalis longicornis. J Vet Med Sci 2008, 70:1363-1366.

48. Franta Z, Frantova H, Konvickova J, Horn M, Sojka D, Mares M, Kopacek P: Dynamics of digestive proteolytic system during blood feeding of the hard tick Ixodes ricinus. Parasit Vectors 2010, 3:119.

49. Anderson JM, Sonenshine DE, Valenzuela JG: Exploring the mialome of ticks: an annotated catalogue of midgut transcripts from the hard tick, Dermacentor variabilis (Acari: Ixodidae). BMC Genomics 2008, 9:552.

50. Santamaria ME, Hernandez-Crespo P, Ortego F, Grbic V, Grbic M, Diaz I, Martinez M: Cysteine peptidases and their inhibitors in Tetranychus urticae: a comparative genomic approach. BMC Genomics 2012, 13:307.

51. Bohley P, Seglen PO: Proteases and proteolysis in the lysosome. Experientia 1992, 48:151-157.

52. Sonenshine DE: Biology of the tick. New York: Oxford University Press; 1991.

53. Coons LB, Alberti G: The acari-ticks. In Microscopic anatomy of invertebrates Volume 8B. Edited by Harrison FW, Foelix RF. New York: Wiley-Liss; 1999:267-514

54. Willadsen P, Riding GA, McKenna RV, Kemp DH, Tellam RL, Nielsen JN, Lahnstein J, Cobon GS, Gough JM: Immunologic control of a parasitic arthropod. Identification of a protective antigen from Boophilus microplus. J Immunol 1989, 143:1346-1351. 
55. Gough JM, Kemp DH: Localization of a low abundance membrane protein (Bm86) on the gut cells of the cattle tick Boophilus microplus by immunogold labeling. J Parasitol 1993, 79:900-907.

56. Willadsen P: Anti-tick vaccines. Parasitology 2004, 129(Suppl):S367-S387.

57. Nuttall PA, Trimnell AR, Kazimirova M, Labuda M: Exposed and concealed antigens as vaccine targets for controlling ticks and tick-borne diseases. Parasite Immunol 2006, 28:155-163.

58. Bartley K, Huntley JF, Wright HW, Nath M, Nisbet AJ: Assessment of Cathepsin D and L-like proteinases of poultry red mite, Dermanyssus gallinae (De Geer), as potential vaccine antigens. Parasitology 2012, 139:755-765.

59. Nisbet AJ, Billingsley PF: A comparative survey of the hydrolytic enzymes of ectoparasitic and free-living mites. Int J Parasitol 2000, 30:19-27.

60. Sikes RK, Chamberlain RW: Laboratory observations on three species of bird mites. J Parasitol 1954, 40:691-697.

61. Shahein YE, Abouelella AM, Hussein NA, Hamed RR, El-Hakim AE, Abdel-Shafy S, Tork SE: Identification of four novel Rhipicephalus annulatus upregulated salivary gland proteins as candidate vaccines. Protein J 2013, 32:392-398.

62. Alarcon-Chaidez FJ, Sun J, Wikel SK: Transcriptome analysis of the salivary glands of Dermacentor andersoni Stiles (Acari: Ixodidae). Insect Biochem Mol Biol 2007, 37:48-71.

63. Valenzuela JG: High-throughput approaches to study salivary proteins and genes from vectors of disease. Insect Biochem Mol Biol 2002, 32:1199-1209.

64. Araki T, Milbrandt J: Ninjurin, a novel adhesion molecule, is induced by nerve injury and promotes axonal growth. Neuron 1996, 17:353-361.

65. De Gregorio E, Spellman PT, Rubin GM, Lemaitre B: Genome-wide analysis of the Drosophila immune response by using oligonucleotide microarrays. Proc Natl Acad Sci USA 2001, 98:12590-12595.

66. Lombardo F, Ghani Y, Kafatos FC, Christophides GK: Comprehensive genetic dissection of the hemocyte immune response in the malaria mosquito Anopheles gambiae. PLoS Pathog 2013, 9:e1003145.

67. Choy RK, Thomas JH: Fluoxetine-resistant mutants in C. elegans define a novel family of transmembrane proteins. Mol Cell 1999, 4:143-152.

68. Choy RK, Kemner JM, Thomas JH: Fluoxetine-resistance genes in Caenorhabditis elegans function in the intestine and may act in drug transport. Genetics 2006, 172:885-892.

69. Dzitoyeva S, Dimitrijevic N, Manev H: Identification of a novel Drosophila gene, beltless, using injectable embryonic and adult RNA interference (RNAi). BMC Genomics 2003, 4:33.

doi:10.1186/1756-3305-6-259

Cite this article as: Schicht et al:: The predicted secretome and transmembranome of the poultry red mite Dermanyssus gallinae. Parasites \& Vectors 2013 6:259.

\section{Submit your next manuscript to BioMed Central and take full advantage of:}

- Convenient online submission

- Thorough peer review

- No space constraints or color figure charges

- Immediate publication on acceptance

- Inclusion in PubMed, CAS, Scopus and Google Scholar

- Research which is freely available for redistribution 